\title{
SEPARATION OF ANISOTROPY AND INHOMOGENEITY \\ INFLUENCE BY THE SPECTRAL ANALYSIS OF AZIMUTHAL RESISTIVITY DIAGRAMS
}

\author{
D.K.Bolshakov, L.N.Modin, E.V.Pervago, V.A.Shevnin (Moscow state university) \\ Russia, 119899, Moscow, MSU Faculty of Geology, fax: (+7-095)-9394963 \\ E-mail: sh@geophys.geol.msu.su
}

The first aim of resistivity survey is a study of any inhomogeneities. Frequently in practice the effect of anisotropy is displayed together with that of layering or inhomogeneities. It complicates data interpretation within the framework of anisotropic models, and distorts results of interpretation in the framework of layered or inhomogeneous media. Ignoring of anisotropy results in wrong data interpretation. At the same time anisotropy studying can give valuable geological information. That means that anisotropy itself and the mutual influence of anisotropy and inhomogeneities needs to be studied. We have developed the effective spectral algorithm for azimuthal resistivity survey (ARS) modeling over two-layered (and multilayered) media with the anisotropic basement. Its advantage is in the fact, that the most labor-consuming part of the decision is carried out in spectral domain, and then transformation of the decision into real domain is fulfilled easily and fast for any azimuths. For two-layered model that approach gives the decision as series, when calculation goes fast and with controllable accuracy. The spectral approach helps to fulfill more deep and full analysis of the decision. The ARS diagram is a periodic function, and has a discrete spectrum. With the help of ARS spectra the important fact was established: for any array above the anisotropic halfspace or horizontally layered medium the odd harmonics are absent in the spectrum. These appear only when the current source is lowered on a depth or the array is placed near some inhomogeneities.

The spectral approach has allowed to explain many facts, established earlier for anisotropic media.

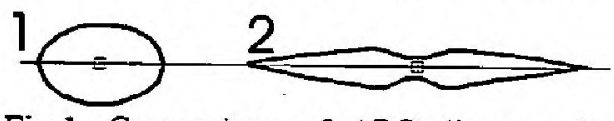

Fig. 1. Comparison of ARS diagram for linear (1) and non-linear (2) arrays over anisotropic halfspace with $\lambda=1.73$

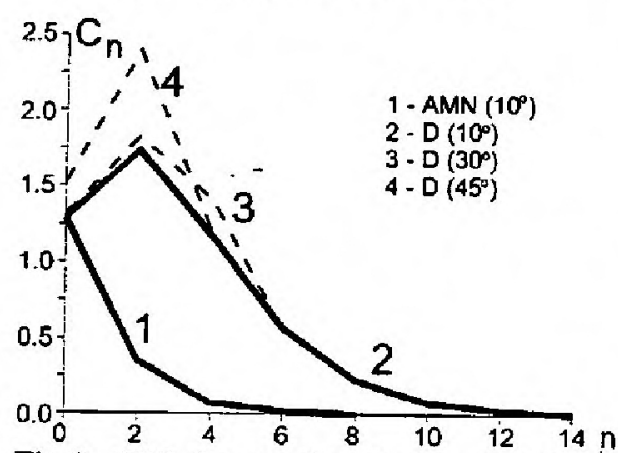

Fig.2. ARS diagrams' spectra for AMN and " $D$ " arrays (even harmonics only)

1. It is known, that linear arrays: $A M(U), A M N(E)$, AMNB (S), dipole axial (A) give the ARS diagrams with the axes ratio $\lambda$, Whereas dipole equatorial (D) array gives $\lambda^{5}$ (fig. 1). Spectra of all linear arrays monotonously decreasing with harmonics, and therefore a zero harmonic has the greatest amplitude. In a spectrum of D array the first even harmonics can be more than zero one and that explains the high sensitivity of dipole equatorial array to anisotropy (fig.2).

2. Fig. 2 shows the results of spectra calculations for theoretical ARS diagrams of pole-dipole and dipole equatorial arrays (with $\mathrm{R} / \mathrm{MN}=10$ ) above an anisotropic half-space where $\rho_{\rho}=1$ and $\rho_{n}=3$. Lines show bending around discrete spectra of only even harmonics (odd ones are equal to zero). The spectrum for AMN is labeled 1, that for "D" by 2; both spectra being calculated for azimuthal steps of $10^{\circ}$. Spectra 3 and 4 refer to the " $\mathrm{D}$ " array where

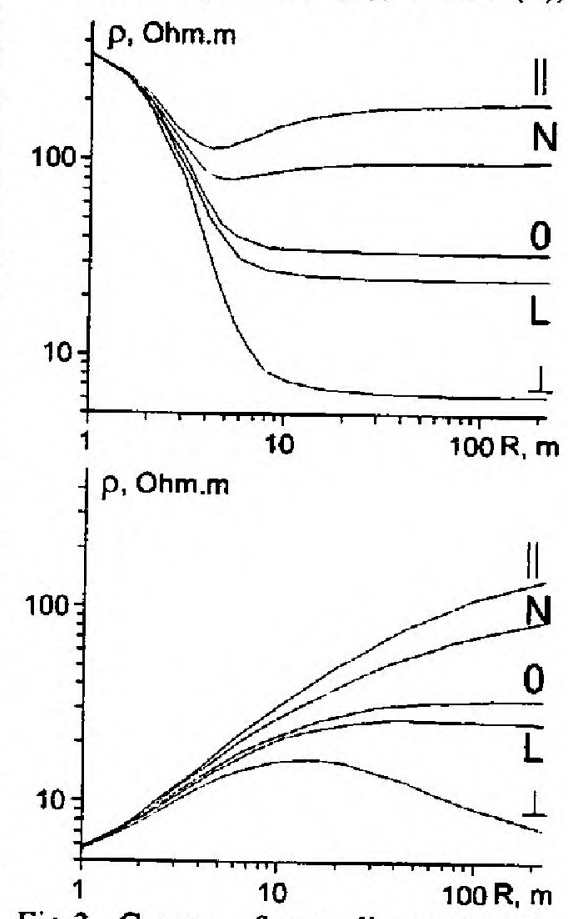

Fig. 3. Curves of sounding with $D$ array and their transformations for two models (sce details in the text) 
calculations have been made in azimuthal steps of $30^{\circ}$ and $45^{\circ}$ respectively and their spectra became distorted. The Kotelnikov - Shennon coding theorem establishes rules for the signal registration without an information loss and can be applied to ARS case also. The width of spectra for any linear array is the same and rather narrow, whereas that for D-array is wider (fig.2). That means, that for ARS measurements with D-array without distortion of its spectnum more azimuths are needed. For linear arrays (U, E, A) an ARS step in $45^{\circ}$ is enough, for $\mathrm{D}$ array with the ratio $\mathrm{R} / \mathrm{MN}=10$ a step in $18-20^{\circ}$ is needed, and for squared array - $\mathrm{H}$ (offered by G.M.Habberjam), where $R M N=1$, the step in $30^{\circ}$ is enough.

3. At the sounding with $D$-array of two-layered model with anisotropic basement the longitudinal and transversal sounding curves are sharply differed on their form. The transversal curve (fig. 3 , curve $\perp$ ) for two-layered model with $\rho_{2}>\rho_{1}\left(\rho_{1}=5, \rho_{2}=25, \rho_{2 n}=100, h_{1}=1\right)$, and the longitudinal curve (fig. 3 , curve $\|$ ) for the model with $\rho_{2}<\rho_{1}\left(\rho_{1}=400, \rho_{2}=25, \rho_{2 n}=100, h_{1}=1\right)$ are looked as three-layered (in the first case as $\mathrm{K}$, and in the second one as $\mathrm{H}$ ) (fig. 3 ).

4. Spectral approach has allowed to simplify the process of interpretation. The iterative process is not required, the parameters can be determined at one step. Together with the azimuth of an anisotropic medium's strike we estimate its error. Instead of interpretation of longitudinal and transversal VES curves, what gives distorted value of the upper layer thickness above the anisotropic basement, we use now dependence of zero harmonic from spacing. allowing to estimate this thickness precisely (fig. 3 , curve 0 ). Spectra allow to transform sounding curves for D-array to linear one, for which the "true" values $\rho_{l}, \rho_{n}, \rho_{m}$ are defined simpler (fig.3, curves $\mathrm{L}, \mathrm{N}$ ). In comparison with direct measurements with linear array, that way gives greater accuracy.

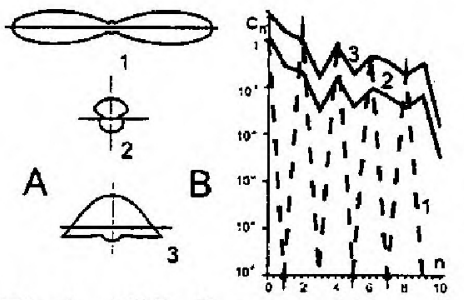

Fig.4. ARS diagrams (A) and their spectra (B) for anisotropic medium (1) and vertical contact $(2,3)$

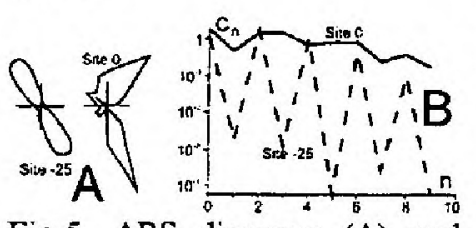

Fig.5. ARS diagrams (A) and their spectra (B) far from (site 25 ) and near to (site 0 ) contact of two anisotropic media

from vertical contact of two anisotropic media consists in presence or absence of odd harmonics (fig.5). One parameter, received from spectra and named a factor of asymmetry $\gamma$ helps to estimate the influence of anisotropy and inhomogeneity. Near to an inhomogeneity it grows, and with removal from it goes to one. $E$ and $D$ arrays have similar sensitivity to inhomogeneities, but quite differently feel anisotropy, that gives a chance to separate these factors with $D$ array.

To see differences in ARS diagrams above anisotropic media and near inhomogeneities the ARS diagrams with azimuth step $10^{\circ}$ for D-array near vertical contact of two media, and also their spectra. and number of additional parameters on the basis of spectra were calculated. Three different models of vertical contact are joint on fig. 6 in one united profile. These are: contact of two isotropic media $(1,2)$ with different resistivities $\left(\rho_{1}=1, \rho_{2}=2\right)$; contact of isotropic and anisotropic media $(2,3)\left(\rho_{m j}=1.5\right.$, $\left.\lambda_{3}=2, \beta_{3}=-20^{\circ}\right)$ : and contact of two anisotropic media with different parameters $(3,4)\left(\rho_{m 4}=3, \lambda_{4}=3, \beta_{4}=45^{\circ}\right)$. For increase of presentation of estimations of azimuths for media 1 and 2 factors of an anisotropy a little bit different from $l$ and azimuth of a strike $\beta=0^{\circ}$ are given. Array spacing $R=2$, $\mathrm{AB}=\mathrm{MN}=0.5$.On fig. 6 results of ARS diagrams processing and interpretation as the graphs of $\rho_{l}, \rho_{m}=\rho_{n}$ (A), factor of an anisotropy $\lambda_{2}$ (B).

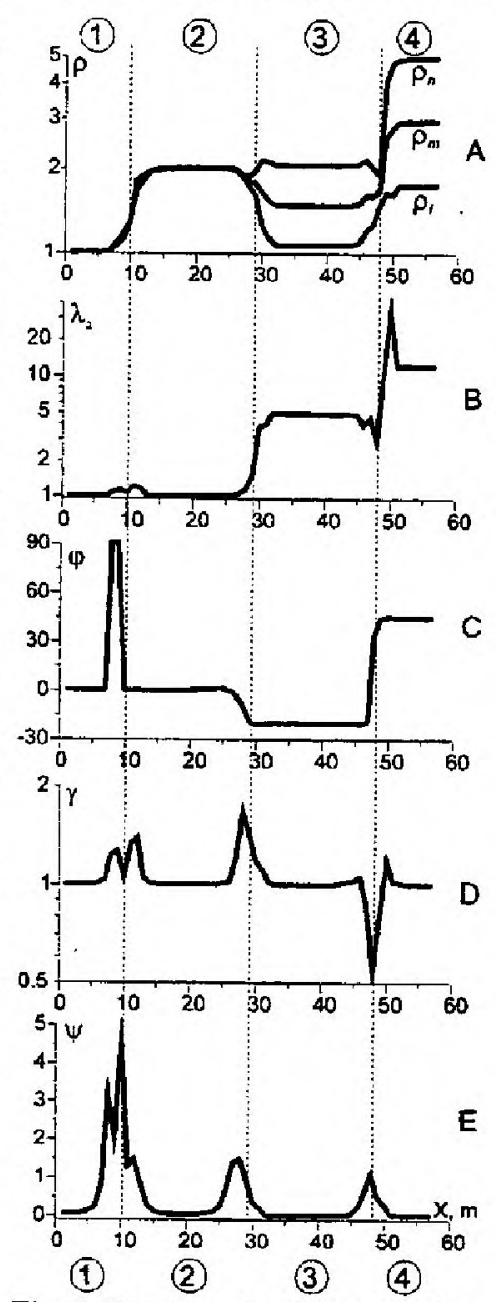

Fig.6. Results of ARS modeling across three vertical contacts with different parameters, united in one profile (see details in text) 


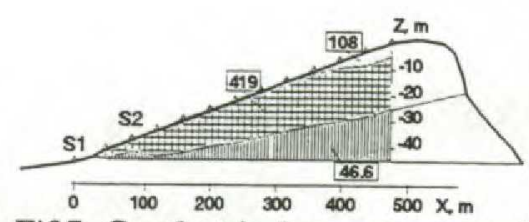

Fif.7. Geoelectrical cross-section of Patil hill along A-A line (see fig. 13). X, Z scales are different.

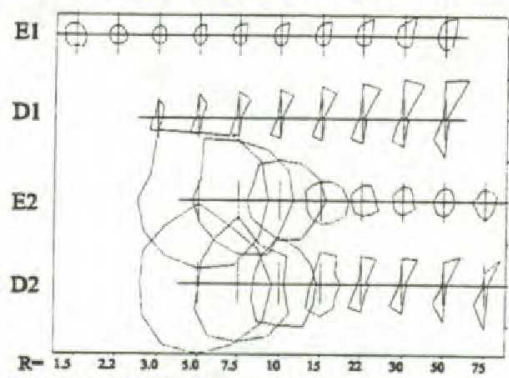

Fig.8. ARS diagrams for $\mathrm{E}$ and $\mathrm{D}$ array in sites $\mathrm{S} 1$ and $\mathrm{S} 2$ depending on spacing $\mathrm{R}$.

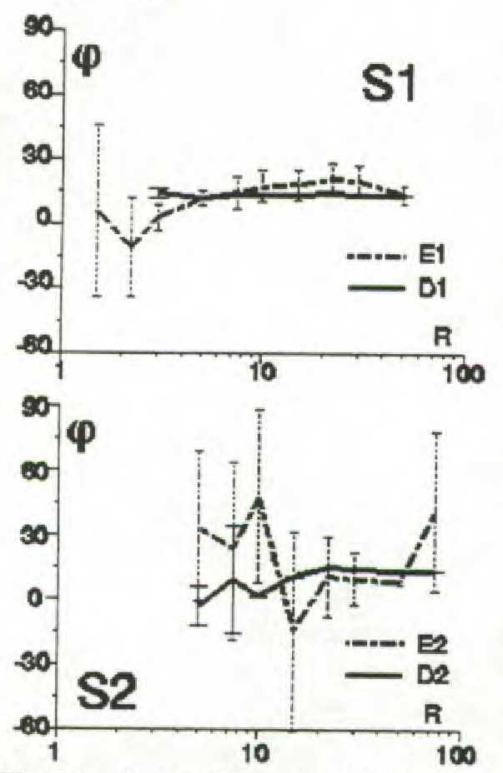

Fig.11. Azimuth estimations and their errors for E and D array in sites 1 and 2 .

(o draw sounding 列 In both cases thè distributions are bimodal and marked by two modal curves drown on fig. 9-10. The upper curve in site 1 corresponds to longitudinal array orientation and looks like two-layered one with $\mu>1$. The lower curve corresponds to transversal array orientation and looks like three-layered H-type curve. Difference between these curves is about ten times in $\rho_{\mathrm{a}}$ level. The curves for S1 show that the medium in this place is not uniform half-space and the reason of that is probably in effect of weathering. VES curves for the site 2 with overburden are also different for longitudinal and transversal directions.

Longitudinal curve looks as descending twolayered one, whereas transversal curve looks as three-layered H-type curve. Theoretical accounts have confirmed, that the difference between both curves and the layer $\mathrm{H}$ on the last curve arises from the anisotropy influence.

The further data processing was made with the help of spectral approach. The basic opportunities of the spectral approach are shown in the other our report,

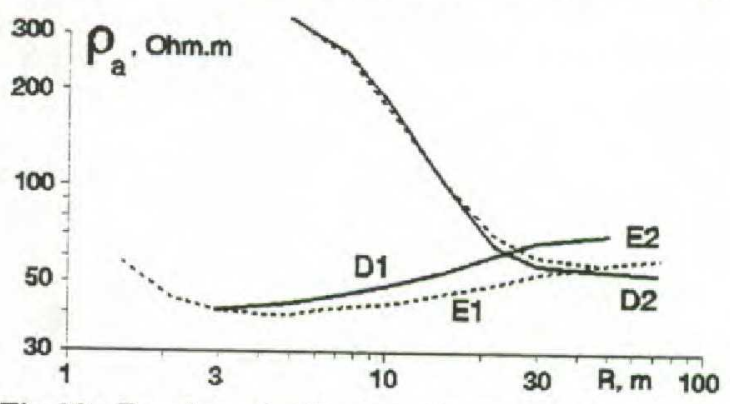

Fig.12. Results of AR soundings transformation into zero harmonic curves. 
submitted on the same conference. Spectral approach allows to define a strike azimuth and an error of that estimation. On fig.11 azimuths' estimations for different spacings and their errors for E and D array are submitted. The error for E array is much more that for D-array. The error for D array is small and frequently is not seen clearly on fig.11.

Fig.12 gives an example of spectral transformation of AR soundings into sounding curves without influence of anisotropy. That can be done with 0harmonics of AR soundings. As we saw on fig.8, ARS diagrams for $\mathrm{E}$ and $\mathrm{D}$ arrays were different, but 0 -harmonics for both arrays on fig. 12 are very similar, and can be interpreted as usual VES curves.

On Fig. 13 azimuthal diagrams for 29 ARS sites on Patil are presented. To the South from line B the anisotropic basement is covered with the layer of sandstone. The sites $\mathrm{T} 0$ and S1 are situated on the outcrop of the basement. The diagrams named T0, S1, S2 and 1-10 were measured with D-array spacing from 20 to $40 \mathrm{~m}$. All other ARS were measured with array spacing from 40 to $120 \mathrm{~m}$. The rotation of Darray was executed around the spacing center. The choice of D-array electrode spacing

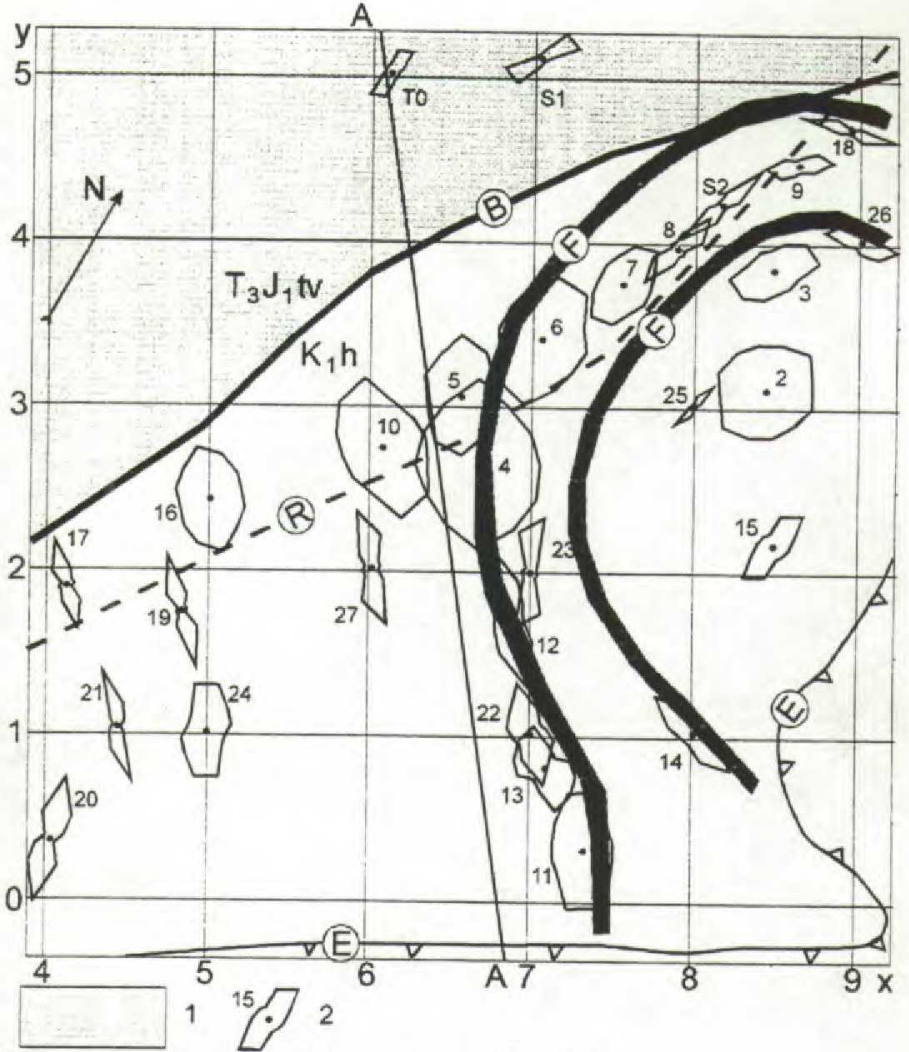

Fig.13. Results of Patil mapping with ARS.

depending on proposed thickness of sandstone cover, was justified with the help of theoretical modeling (Bolshakov et al. 1995). The difference in the shape of the AR diagrams S2, 8, 7, 6, 5, 10,4 result from the gradual increase in the thickness of the upper layer. The difference in the shape of AR diagrams 2 and 25 is a result of a change in array spacing (40 and $120 \mathrm{~m})$.

Delineation of the strike in the basement rocks with AR diagrams helped to map a fold, situated in the right part of fig. 13. The form of the fold is marked by lines with the letter F. The abrupt edge of Patil hill in the South and the East is marked by a line with letter E. Line A-A is VES profile (see results on fig.7).

\section{CONCLUSIONS}

The dipole equatorial array (D) is much more sensitive to the anisotropy of a medium, than any linear array. That allows to estimate more stable anisotropic parameters, even in case of an anisotropic medium covered with overburden. D - array needs a more detailed azimuthal step in ARD measurements $\left(15-20^{\circ}\right)$, than any linear array.

In anisotropic media where inhomogeneities are present, D - array, unlike a linear array, becomes more sensitive to anisotropy without an appreciable change in sensitivity for the identification of inhomogeneities. Hence there is an opportunity of distinguishing anisotropy effects from the influence of inhomogeneities.

The analysis of ARS results with the help of decomposition in Fourier series is an effective tool for studying anisotropic and inhomogeneous media, and provides an instrument of anisotropy and inhomogeneities comparative estimation. Ideally, identification of inhomogeneities and anisotropy influences is desirable to fulfill with not one ARD measurement, but in a series of measurements either along profiles or throughout the area under investigation. We recommend non-symmetrical rotation of arrays (for example, to rotate the D - array around the center of $A B$ (or $M N$ ), instead of spacing center).

Azimuthal measurements with the dipole equatorial array can confidently estimate the strike of an anisotropic formation, including the case when the anisotropic basement is covered with overburden.

\section{REFERENCES}

1. D.K.Bolshakov, I.N.Modin, E.V.Pervago, V.A.Shevnin. Anisotropy effects investigations by resistivity method in some inhomogeneous media. Extended abstracts of EAEG 57th Annual Meeting, Glasgow, May 28 - June 2, 1995. P034

2. D.K.Bolshakov, I.N.Modin, E.V.Pervago, V.A.Shevnin. Study of VES peculiarities over anisotropic basement with overburden. Vestnik of MSU, geol. ser., 1996, N 2, pp.60-70. (In Russian).

3. A.S.Semenov. Rock anisotropy and electrical fields peculiarities in anisotropic media. Vestnik of Leningrad university, ser. Geol. and geography. 1975. N 24. p.40-47 (In Russian).

4. Electrical prospecting by resistivity method. Editors: V.K.Khmelevskoj and V.A.Shevnin. MSU edition, Moscow, 1994, 160 pp. (In Russian)

5. G.M.Habberjam. The effect of anisotropy on square array resistivity measurements. Geophysical Prospecting 1972,20, N 2, p. $249-266$ 